\section{Therapeutic innovation for multi-resistant candidemics: Synergy of isavuconazole and caspofungin association}

\author{
Andrea Duminuco, ${ }^{1}$ Elisa Mauro, ${ }^{2}$ \\ Giuseppe A. M. Palumbo, ${ }^{2}$ \\ Bruno Garibaldi, ${ }^{1}$ Marina Parisi, ${ }^{2}$ \\ Francesco Di Raimondo, ${ }^{2}$ \\ Cinzia Maugeri, ${ }^{2}$ Calogero Vetro ${ }^{2}$ \\ ${ }^{1}$ Post Graduation School of Hematology, \\ University of Catania, A.O.U. \\ 'Policlinico-San Marco', Catania; \\ ${ }^{2}$ Division of Hematology, A.O.U. \\ 'Policlinico-San Marco', Catania, Italy
}

\section{Introduction}

Fungal infections are an emerging problem in immunocompromised patients with hematological diseases. Fungal organisms such as Candida glabrata or tropicalis are becoming increasingly important in these areas, as they are increasingly able to acquire resistance to the common drugs and lead to the death of such patients. In this case report, we describe the excellent results obtained with the experimental combination of two antifungal agents (isavuconazole and caspofungin) in the treatment of an acute myeloid leukemia patient with disseminated Candida infection. This association is based on recent evidence (especially in vitro studies) of synergy of action against agents such as Aspergillus and Candida auris.

\footnotetext{
Abstract

Fungal infections occurring in immunocompromised patients after immunochemotherapy treatment are often difficult to eradicate and capable of even being fatal. Systemic mycoses affecting severely immunocompromised patients often manifest acutely with rapidly progressive pneumonia, fungemia, or manifestations of extrapulmonary dissemination. Opportunistic fungal infections (mycoses) include several pathogens elements, as candidiasis, aspergillosis, mucormycosis (zygomycosis) and fusariosis. Prompt diagnosis and effective therapy are needed to improve the associated morbidity and mortality, especially in cases with non-canonical fungal localizations and not responsive to the available antifungal drugs.
}

\section{Case Report}

We report a case of a 46-years-old-male patient affected by secondary acute myeloid leukemia (sAML), arisen from previous myelodysplastic syndrome (MDS) and treated with CPX-351 (liposomal formulation of daunorubicin and cytarabine). ${ }^{1}$ To reduce the risk of fungal infection due to profound aplasia, the patient received antifungal prophylaxis with posaconazole. ${ }^{2}$ This drug, however, was taken irregularly and not continuously, cause low compliance of the patient. During post-chemotherapy pancytopenia, the clinical course was characterized by infectious complications, with several fever peaks per day accompanied by presence of shaking chills. C-reactive protein (CRP) was elevated $(388.33 \mathrm{mg} / \mathrm{L}$, normal values $0-5 \mathrm{mg} / \mathrm{L})$. Chest computed tomography (CT) was performed to search for any source of infection, finding multiple small parenchymal thickenings with a micronodular appearance, disseminated in apical and posterior basal segments. The patient was treated with different lines of empirical antibiotic therapy, without success. From peripheral vein blood cultures, performed during a febrile peak, candida tropicalis, sensitive to caspofungin, micafungin, voriconazole and anidulafungin, was isolated and, on blood chemistry tests, the value of 1-3- $\beta$-glucan (polysaccharide essential for the formation of fungal cell wall and directly proportional to fungal infection) was elevated $(220 \mathrm{pg} / \mathrm{mL}$, normal values $<80 \mathrm{pg} / \mathrm{mL}$ ). ${ }^{3}$ The patient was unsuccessfully treated with several lines of single agent antifungal drugs: caspofungin 50 $\mathrm{mg}$ /die for one week, voriconazole $200 \mathrm{mg}$ bid for two weeks, fluconazole $400 \mathrm{mg} / \mathrm{die}$ for 10 days and amphotericin-B $240 \mathrm{mg}$ for 3 weeks. Given the poor response to these therapies, the clinic's worsening and the lung infection to instrumental examinations ('pulmonary inflammation spread over all lobes, with prevalent interstitial involvement, micronodular pattern and groundglass level' on re-evaluation CT) (Figure 1A), the patient underwent to a bronchoalveolar lavage (BAL) procedure, where the presence of candida tropicalis, candida glabrata and pneumocystis jirovecii was found. At the same time, the patient began to complain about progressive vision reduction in both eyes, especially in left eye. The patient underwent fundus oculi examination, where the presence of retroioloid haemorrhages at the posterior pole of both eyes and a rounded foveal cotton exudate in the left eye was found. These images, analyzed in depth with fluorangiography, were very suspicious for fungal infection due to
Correspondence: Andrea Duminuco, PostGraduation School of Hematology, University of Catania, A.O.U. 'Policlinico-San Marco', via S. Sofia 78, 95123, Catania, Italy.

Tel. +39.0953.781997. Fax: +39.0953 .782977 .

E-mail: andrea.duminuco@gmail.com.

Key words: Fungal infection, Candida, Isavuconazole, Caspofungin, Hematological patients, Combined antifungal therapy.

Contributions: All the authors have read and approved the final version of the manuscript and agreed to be accountable for all aspects of the work.

Conflict of interest: The authors declare no potential conflict of interest.

Funding: None.

Ethics approval and consent to participate: No ethical committee approval was required for this case report by the Department, because this article does not contain any studies with human participants or animals. Informed consent was obtained from the patient included in this study.

Consent for publication: The patient gave his written consent to use his personal data for the publication of this case report and any accompanying images.

Availability of data and materials: All data generated or analyzed during this study are included in this published article.

Received for publication: 22 July 2021.

Revision received: 2 September 2021.

Accepted for publication: 17 September 2021.

This work is licensed under a Creative Commons Attribution-NonCommercial 4.0 International License (CC BY-NC 4.0).

(C) Copyright: the Author(s), 2021

Licensee PAGEPress, Italy

Hematology Reports 2021; 13:9329

doi:10.4081/hr.2021.9329

their chorioretinal localization due to candida (Figure 1B), a potentially dangerous condition if not properly treated, as it causes extensive vitreous inflammation and possible loss of the eye. ${ }^{4-6}$

Despite the conventional antifungal drugs, the values of 1-3- $\beta$-D-glucan and CRP remained persistently elevated and febrile episodes continued to be present. We therefore decided to treat the patient with combination of caspofungin $70 \mathrm{mg}$ /day in association with isavuconazole $200 \mathrm{mg}$ x 3 /day. ${ }^{4-6}$ This therapy was continued for a month, with progressive improvement of 

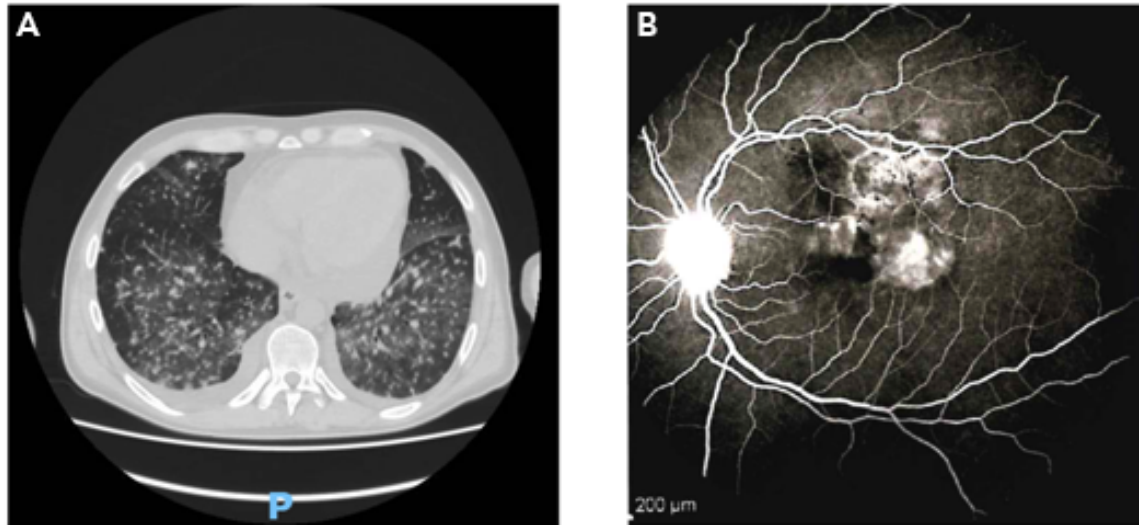

Figure 1. Evidence of mycotic localization in lungs (A) and eye (B), as described in text.

symptoms and a notable reduction in the values of $1-3-\beta$-glucan $(90 \mathrm{pg} / \mathrm{mL})$, before total recovery of blood count values. For ocular candida's location, our therapeutic choice was intravitreal injection of 100 $\mathrm{ug} / 0.1 \mathrm{~mL}$ of off-label voriconazole, based on experimental studies in the literature. ${ }^{7-9}$ After a single administration of voriconazole, completed without any noteworthy side effects, there was a notable clinical improvement with gradual vision recovery. Continuing the combination therapy between the two antifungals, the febrile episodes disappeared and the value of 1-3$\beta$-glucan reduced to the point of negativization in four consecutive measurements $(<80 \mathrm{pg} / \mathrm{mL})$. Thus, maintaining the complete remission from the haematological disease reached after chemotherapy and treating the infection given by candida with the combination of isavuonazole and caspofungine and the chorioretinal localization of the same mycete with the intravitreal administration of voriconazole, the patient was able to complete his therapeutic process, undergoing then a bone marrow transplant, having a complete blasts clearance.

\section{Discussion And Conclusions}

A prompt diagnosis of fungal infection is crucial in dealing with immunosuppressed haemato-oncological patients. In cases of infections not responsive to main antifungals, it is important to look for any uncommon localization. In our experience, the localization of candida at the ocular level has been demonstrated, successfully treated with off-label intravitreal of voriconazole. Instead, the real challenge lies in the management of cases of infection not responsive to the administration of available drugs: there are little data of candida's infections treated with antifungals in combination with each other. In particular, to the best of our knowledge, the only study about the combination between isavuconazole and caspofungin in literature is an in vitro analysis regarding the synergy between these two antifungals against aspergillus spp, where the results are not particularly suggestive of a possible strengthening of their mechanisms of action, ${ }^{10}$ and a very recent analysis of their interaction with candida auris, showing synergy of action both in vitro and in vivo tests, corresponding to safe drug concentrations and clinically achievable. ${ }^{11}$ This combination therapy, in our case, had considerable success, considering also that these drugs in single administration have not been able to obtain the desired results, allowing the patient to continue and complete his life-saving therapeutic process. Starting from this drug association, future studies are necessary to confirm the prospect of validity of this interesting therapeutic hypothesis in multidrug-resistant fungal infections.

\section{References}

1. Lancet JE, Uy GL, Cortes JE, et al. Final results of a phase III randomized trial of CPX-351 versus $7+3$ in older patients with newly diagnosed high risk (secondary) AML. J Clin Oncol 2016; 34:7000.

2. Girmenia C, Frustaci AM, Gentile G, et al. Posaconazole prophylaxis during front-line chemotherapy of acute myeloid leukemia: a single-center, reallife experience. Haematologica 2012; 97:560-67.

3. Racil Z, Kocmanovà I, Winterovà $J$, et al. Detection of 1,3-beta-D glucan for diagnosis of invasive fungal infections in hematooncological patients: usefulness for screening of invasive mycosis and for confirmation of galactomannan positive results. Klin Mikrobiol Infekc Lek 2009; 15:48-57.

4. Lingappan A, Wykoff CC. Albini TA, et al. Endogenous fungal endophthalmitis: causative organisms, management strategies, and visual acuity outcomes. Am J Ophthalmol 2012;153:162-6.

5. Danielescu C, Anton N, Stanca HT, Munteanu M. Endogenous endophthalmitis: a review of case series published between 2011 and 2020. Journal of Ophthalmology 2020;2020:8869590.

6. Shah CP, McKey J, Spirn MJ, Maguire J. Ocular candidiasis: a review. British Journal of Ophthalmology 2008;92: 466-8.

7. Karagoz E, Ugan RA, Duzgun E, et al. A comparative study of the effects of intravitreal Anidulafungin, Voriconazole, and Amphotericin B in an experimental candida endophthalmitis model. Curr Eye Res 2017;42:225-32.

8. Khan FA, Slain D, Khakoo RA. Candida endophthalmitis: focus on current and future antifungal treatment options. Pharmacotherapy 2007;27: 1711-21.

9. Bienvenu AL, Aussedat M, Mathis T, et al. Intravitreal injections of voriconazole for candida endophthalmitis: a case series. Ocu Immunol Inflam. 2020;28: 471-78

10. Raffetin A, Courbin V, Jullien V, Dannaoui E. In vitro combination of isavuconazole with echinocandins against azole-susceptible and -resistant aspergillus spp. Antimicrob Agents Chemother 2017;62:e01382-17.

11. Nagy F, Tòth Z, Nyikos F, et al. In vitro and in vivo interaction of caspofungin with isavuconazole against candida auris planktonic cells and biofilms. bioRxiv 2021;59:1015-23. 